\title{
Serial Section Scanning Electron Microscopy of Adult Brain Tissue Using Focused Ion Beam Milling
}

\author{
Graham Knott, ${ }^{1}$ Herschel Marchman, ${ }^{3}$ David Wall, ${ }^{2}$ and Ben Lich ${ }^{2}$ \\ ${ }^{1}$ Centre Interdisciplinaire de Microscopie Electronique, Ecole Polytechnique Federale de Lausanne, 1015 Lausanne, Switzerland, ${ }^{2}$ FEI Company, Acht, 5651 \\ GG Eindhoven, The Netherlands, and ${ }^{3}$ Janelia Farm Research Campus, Ashburn, Virginia 20147
}

\section{Introduction}

Analyzing the synaptic basis of neuronal circuits within a volume of brain tissue requires electron microscopy. With a resolution capable of seeing the smallest synaptic contacts, this method uses different sectioning techniques to produce serial images suitable for seeing the ultrastructure within significant volumes of brain tissue. Sections are cut from a block of tissue, leaving either the block face to be imaged with a scanning electron microscope or the section itself to be imaged with a with a transmission electron microscope. Here, we have explored the use of focused ion beam milling combined with block-face scanning electron microscopy to serially cut and image a volume of neuropil in the adult rodent brain. We show that a conventional transmission electron microscopy fixation, staining, and embedding procedure gives sufficient membrane contrast to see all the axons and dendrites and their synaptic contacts. Milling and imaging of the block face provided serial images with sufficient resolution to allow the observer to follow all the neurites and their connections throughout the volume. This technology provides a viable alternative to conventional tissue

Received July 13, 2007; revised Jan. 24, 2008; accepted Feb. 2, 2008.

This work was supported by Swiss National Science Foundation Grant 3100A0-112335/1 (G.K.). We thank Egbert Welker, Michael Hausser, and Arnd Roth for their helpful comments during the preparation of this manuscript and Olivier Wilhelmi for his technical assistance.

Correspondence should be addressed to Graham Knott, Centre Interdisciplinaire de Microscopie Electronique, Ecole Polytechnique Federale de Lausanne, 1015 Lausanne, Switzerland. E-mail: Graham.Knott@effl.ch.

DOI:10.1523/JNEUROSCI.3189-07.2008

Copyright $\odot 2008$ Society for Neuroscience $\quad 0270-6474 / 08 / 282959-06 \$ 15.00 / 0$ slicing and EM imaging approaches for the acquisition of large datasets that could be used for the reconstruction and analysis of neural connectivity.

For several decades, electron microscopy has been used to study neuronal ultrastructure, from the first study using transmission electron microscopy (TEM) to classify synapses in the cerebral cortex (Gray, 1959) to detailed serial section analyses on large parts of identified neurons (White and Rock, 1980; Anderson et al., 1994). Despite advances in light microscopy, only electron microscopy is capable of seeing every axon, dendrite, and synaptic connection within a volume of neuropil. However, imaging large volumes of tissue with serial section transmission EM is one approach that, for the moment, relies on considerable manual labor by skilled persons. Studies thus far have analyzed either the connectivity within tiny portions of complex nervous systems (Shepherd and Harris, 1998) or complete systems of much simpler ones (White et al., 1986). Successful acquisition of large sets of aligned TEM images is challenging for even the experienced microscopist. A number of factors can contribute to interrupted series or imperfect image stacks: section folds during the placement onto support films, electron beam damage that distorts the sections, and stain contamination.

Recently, an alternative to the serial section TEM approach for imaging tissue was described using scanning electron microscopy. A resin-embedded tissue sample was serially sectioned, using a mic- rotome integrated inside a scanning electron microscope (SEM) (Denk and Horstmann, 2004), and the milled face was imaged with the scanning electron beam. The method therefore relies on the mechanical microtomy approach, with a diamond knife cutting thin sections from the block face. Unlike TEM, in which the contrast in the images is generated by the elastic scattering of electrons in regions of higher density, the block-face scanning approach captures electrons backscattered from below the surface of the block itself. More backscattering occurs in regions of higher electron-dense stain, making it possible to image conventionally prepared biological tissue. This technique can be highly automated, removing many of the problems associated with manual serial section manipulation and imaging in the TEM, automatically generating aligned serial images.

We present here an alternative sectioning approach using a focused ion beam (FIB) directed parallel to a block face for removing (or milling) thin layers of embedded tissue, and a scanning electron beam integrated into the same microscope for imaging this milled region (Heymann et al., 2006). We used this technology to serially image conventionally prepared adult brain tissue at a resolution that allowed us to follow the axons and dendrites in a volume of $286 \mu \mathrm{m}^{3}$, and identify their synaptic connections. This milling approach can remove layers as thin as $15 \mathrm{~nm}$ from the block face. The study demonstrates the potential of this method for the automated collection of 
serial images through volumes of brain tissue that can be used for the analysis of neural connectivity.

\section{Materials and Methods}

\section{Tissue preparation}

An adult mouse (female, C57BL/6; 10 weeks of age) was deeply anesthetized using an intraperitoneal injection of sodium pentobarbitone. The animal then underwent chemical fixation by cardiac perfusion of $10 \mathrm{ml}$ of isotonic PBS followed immediately with $200 \mathrm{ml}$ of $2.5 \%$ glutaraldehyde and 2\% paraformaldehyde in phosphate buffer $(0.1 \mathrm{M}, \mathrm{pH} 7.4)$. Two hours after perfusion, the brain was then removed, and 60 - $\mu \mathrm{m}$-thick vibratome (Leica VT100; Leica Microsystems, Wetzlar, Germany) sections were cut through the somatosensory cortex and the cerebellum. The sections were then washed in cacodylate buffer $(0.1 \mathrm{M}, \mathrm{pH} 7.4)$ and postfixed for $40 \mathrm{~min}$ in $1.5 \%$ potassium ferrocyanide and 1\% osmium tetroxide, followed by $1 \mathrm{~h}$ in $1 \%$ osmium tetroxide alone, each in the same buffer, and then 40 min in $1 \%$ uranyl acetate in water. They were then dehydrated in alcohol and finally infiltrated with Durcupan resin (Fluka, Buchs, Switzerland). The sections were flat embedded between glass slides in fresh resin and left for $48 \mathrm{~h}$ at $60^{\circ} \mathrm{C}$ for the resin to harden. This procedure was in accordance with the Swiss Federal Laws on Animal Experimentation administered by the Véterinaire Cantonale Lausanne.

\section{Block preparation}

Once the resin was hard, a $1 \times 1 \mathrm{~mm}$ square block was cut from a section that contained layer IV of the somatosensory cortex. This block was stuck to a blank resin support, and using a microtome and glass knife, trimmed down to a block face measuring $600 \times 200 \mu \mathrm{m}$ (Fig. 1). These are typically the block dimensions used to make serial sections for TEM. The final block was then mounted, using conductive silver paint (Electron Microscopy Sciences, Hatfield, PA), on a standard SEM microscope stub, and then coated with a layer of platinum/palladium (thickness of $\sim 30 \mathrm{~nm}$ ) using a sputter coater (Cressington Scientific, Watford, UK) to remove any charge that may build up on the surface.

The block was placed inside a DualBeam microscope (FEI Helios NanoLab; FEI Company, Acht, Eindhoven, The Netherlands), which combines a scanning electron beam produced by a field emission gun, with a focused beam of gallium ions. This ion beam was used in conjunc-
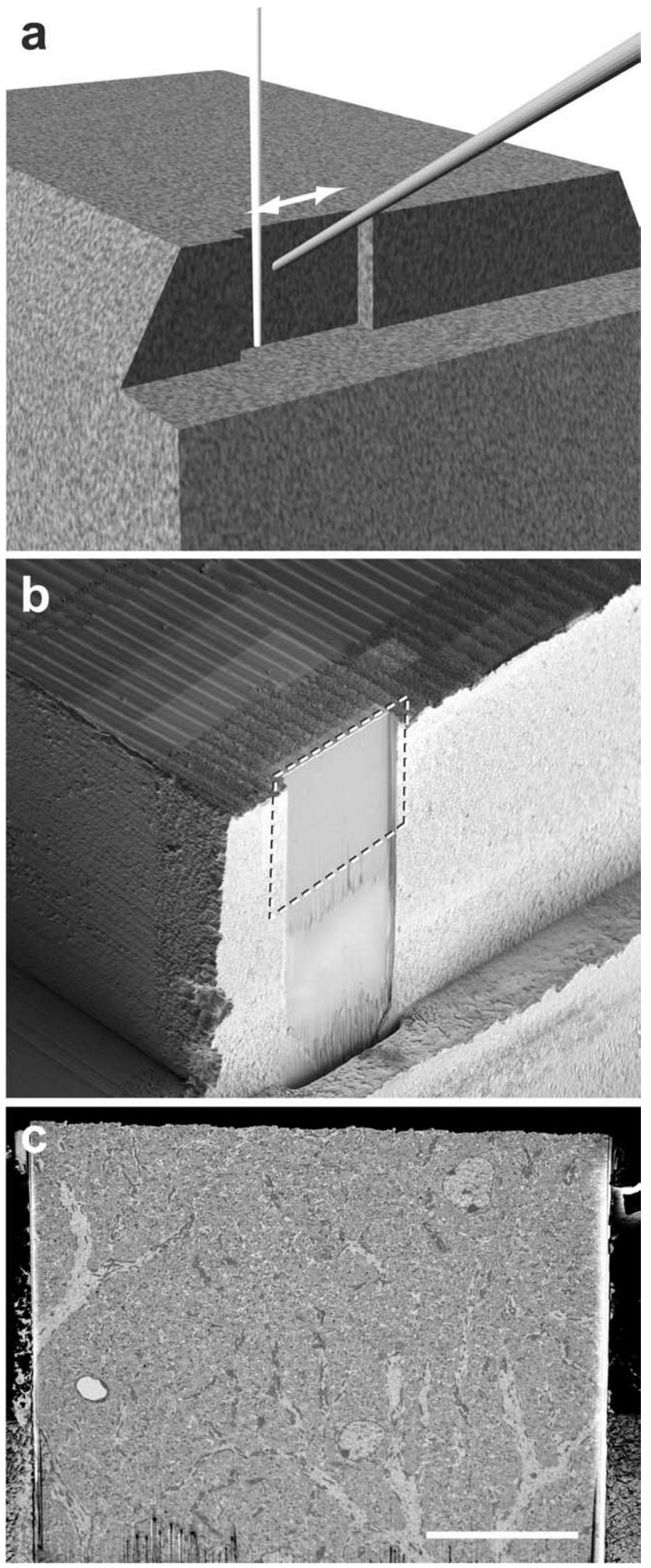

Figure 1. a, Three-dimensional drawing of the resin block showing the arrangement of the gallium ion beam (white) that scans parallel to the block (indicated by double-headed arrow) and mills away a layer of resin, creating an imaging face. The electron imaging beam (gray) at an angle of $52^{\circ}$ from this vertical face is then used to image the embedded tissue at this surface. $\boldsymbol{b}$, Scanning electron micrograph of a tissue block in the same orientation as $\boldsymbol{a}$ with a dotted line indicating the milled region, which is shown in cin reverse contrast. Scale bar, $20 \mu \mathrm{m}$.

tion with a gas injection system to deposit a thick layer $(\sim 1 \mu \mathrm{m})$ of platinum on the top surface of the sample, above the region of interest. This system releases a pre- cursor gas, methylcyclopentadienyl (trimethyl) platinum (IV) (FEI Company), close to the sample. When the gallium ions interact with the sample, in the pres- 
ence of this gas, decomposition takes place, causing platinum solid to be deposited on the surface of the sample where the ion beam was directed.

This additional deposition of a platinum layer immediately above the region of interest reduced FIB milling artifacts. The coating ensured that the resin face was milled evenly and remained parallel to the direction of ion beam. Without it, excessive nonuniform milling occurred because of sample topography or material differences in the face. This can lead to excessive streaking or vertical stripes down the block face known as curtaining (Fig. 2a) (Heymann et al., 2006).

\section{Sectioning and imaging}

To prepare the initial imaging face, a region measuring $\sim 10,000 \mu \mathrm{m}^{2}$ (Figs. $1 c$, 2a) was milled. Essentially, the beam skimmed the surface, and when ions collided with molecules in the material they were ejected away. As the beam scans parallel to the block face, it is slowly moved closer and closer until a $100 \times 100 \mu \mathrm{m}$ face had been milled and all the resin in the path of the ion beam removed. This created a smooth, polished face parallel to the plane of milling (Fig. 1). The progress was monitored using images formed from the electron beam, simultaneous with the ion beam milling [simultaneous patterning and imaging (SPI) mode]. Next, the ion beam was set to mill layers from this region. We milled $40 \mathrm{~nm}$ layers, but also tested milling as little as $15 \mathrm{~nm}$. After each layer was removed, a backscattered electron image was collected. The electron imaging beam is directed at an angle of $52^{\circ}$ to the block face (Fig. 1a), the image being generated through the electrons interacting with the sample and being backscattered out again. These electrons were detected via the in-lens detector, using a negative potential on the tube through which the electrons were collected. This negative potential meant that the lowerenergy secondary electrons were repulsed with only the high-energy backscattered electrons contributing to image formation.

Image interference can occur when milled material redeposits around the imaged area. When this occurs, there is a risk that it can build up and obscure the field of view. For this reason, the milled face was always much larger than the imaged area in both the $x$ and $y$ direction, to ensure that redeposition did not occur nearby.

From the initial $100 \times 100 \mu \mathrm{m}$ face, a smaller region was selected, measuring
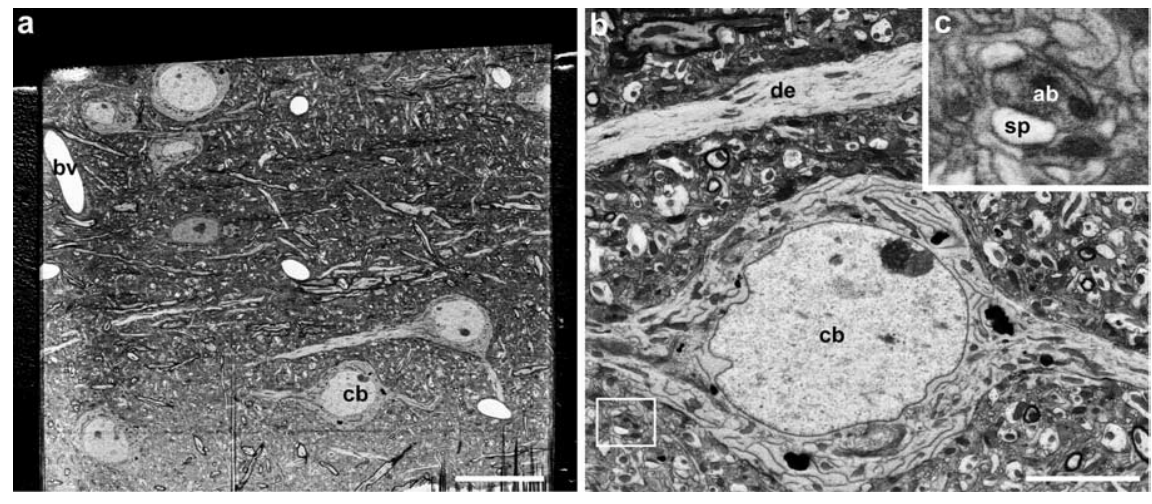

Figure 2. $\quad \boldsymbol{a}$, Reverse-contrast backscattered electron micrograph of an imaging face milled to expose a field of view $\sim 120 \times$ $115 \mu \mathrm{m}$. For this initial preparation phase, a small amount of "curtaining" can be seen in the bottom right corner. This imaging artifact can be removed once serial milling and imaging begins. The image was taken with a beam voltage of $5 \mathrm{keV}$, beam current of $0.40 \mathrm{nA}$, and pixel dwell time of $10 \mu \mathrm{s}$. The resolution of the image is $30 \mathrm{~nm} /$ pixel. Within this image, cell bodies (cb) and blood vessels (bv) are easily visible. $\boldsymbol{b}$, Reverse-contrast backscattered scanning electron micrograph taken from the imaging face shown in $\boldsymbol{a}$, of the neuronal cell body indicated as cb, using the same beam parameters, but a resolution of $10 \mathrm{~nm} / \mathrm{pixel}$. With these settings, the microscope can generate images that clearly show the stained membranes and many features within the neuropil, e.g., dendrites (de). $\boldsymbol{c}$, The same image of the region enclosed in the white square in $\boldsymbol{b}$, which has been enlarged to illustrate the detail that can be seen with these imaging parameters. The image shows an axonal bouton $(\mathrm{ab})$ and dendritic spine head (sp). Scale bars: $\boldsymbol{a}, 20 \mu \mathrm{m} ; \boldsymbol{b}, 5 \mu \mathrm{m}$.

$\sim 30 \times 30 \mu \mathrm{m}$, free from blood vessels and not completely obscured by cell somata, therefore containing mostly axons, dendrites, and astrocytic processes. This smaller face was milled, using an acceleration voltage of $30 \mathrm{keV}$ and a current of 1000 pA. Each mill took 75 s to complete. After the removal of each layer, images were collected from a smaller subregion at a magnification of $4 \mathrm{~nm} /$ pixel, and with a total image size of $2048 \times 1768$ pixels $(\sim 4$ $\mathrm{MB} /$ image). This gave a horizontal field width of $8.2 \mu \mathrm{m}$. The acceleration voltage of the imaging beam was $2 \mathrm{keV}$, with a current of between 340 and 400 pA and dwell time of $100 \mu \mathrm{s} /$ pixel (unless otherwise indicated). For each image collected, the contrast was adjusted automatically. Throughout the entire procedure, the ion and electron beam-coincident position remained at $5 \mathrm{~mm}$ from the final lens of the SEM column.

Although SPI mode was used to monitor the creation of the block face, the acquisition of the higher-resolution images was performed after the milling had finished. Milling and acquisition was fully automated, producing a series of nearly aligned images. Final alignment was achieved manually using Photoshop software (Adobe Systems, San Jose, CA). Individual images were overlaid on the preceding one, as a separate image, in a single file. Optimal alignment was achieved by making the top image (layer) partially transparent and moving it in the $x$ and $y$ direction until it was aligned with the visible image (layer) immediately below. Realignment of the serial images was never
$>40$ pixels in the $x$ or $y$ direction, corresponding to $0.16 \mu \mathrm{m}$. The contrast of the images was also reversed to give the final images the more familiar TEM look.

\section{Results}

Because the images were generated from the backscattered electrons, we wanted to estimate the depth that they were penetrating. This volume of interaction is a function of the density of the sample and the accelerating voltage. The lower the accelerating voltage, the less penetrating the electron beam, therefore, the greater the information that is obtained close to the surface (Denk and Horstmann, 2004). This will have a significant effect on the imaging resolution, such that lower accelerating voltages will provide higher effective resolution, which may be critical for resolving small structures such as membranes and vesicles. Therefore, different acceleration voltages were tested to identify the appropriate voltage for identifying the cell membranes and synapses. Because we made $40 \mathrm{~nm}$ slices, an electron beam energy was selected that would give backscattered electrons at a depth that is less than this slice thickness (Fig. 3).

The acceleration voltage of $2 \mathrm{keV}$ allowed us to see membranes, including those of vesicles within axonal boutons. At higher voltages $(5 \mathrm{keV})$, these features were less distinct, and deeper underlying structures blurred the images. This is particularly evident when a myelinated axon, lying beneath the surface, appears as a shadow in part of the image (Fig. 3a). This shadowing does not appear when a lower 
voltage (2 keV) is used (Fig. $3 b$ ), reducing the interaction volume to what would appear to be less than the slice thickness.

We tried to estimate the depth from which the electrons were being backscattered using $2 \mathrm{keV}$ beam energy. Using a resin block of cerebellar tissue, we imaged the molecular layer, examining a single asymmetric synapse and its vesicles. We used the same imaging parameters as previously. The face was milled at depths of $15 \mathrm{~nm}$, and in each image vesicles could clearly be identified. Because these structures have diameters of between 40 and 50 $\mathrm{nm}$, when section thickness is $15 \mathrm{~nm}$, they can be sectioned a maximum of four times. We saw in our image series that each vesicle was present in three to four images as a circular membrane with a clear core (supplemental Fig. 1, available at www.jneurosci.org as supplemental material). However, they first appear in a single preceding image, but without a clear center, suggesting that the imaging beam penetrates to a depth below the surface at which backscattering occurs from the vesicle. Because this occurs only once before it appears as a hollow structure, presumably when it is imaged at the surface, it would indicate a penetration depth of $<30 \mathrm{~nm}$.

In our larger imaging series taken from the neocortex, we milled 120 layers, each $40 \mathrm{~nm}$ thick, from the block face. This entire process of repeated milling and imaging occurred without any intervention from the operator. Each image was $2048 \times 1768$ pixels with a pixel dwell time of $100 \mu \mathrm{s}$; therefore, each image took $\sim 4$ min to acquire. A subset of the image series is shown as supplemental data (supplemental movie 1, available at www. jneurosci.org as supplemental material) in which a portion of the field of view has been included (Fig. $4 c$, region indicated by dotted line). This shows the milling and imaging process, enabling the viewer to follow each individual neurite through the tissue.

To independently check the milling thickness, we used the method of Fiala and Harris (2001). Measuring the diameter of several mitochondria lying longitudinal to the imaging plane and counting the number of times this region was cut allows us to make an estimate of section thickness, presuming these structure are cylindrical. For the series cut at $40 \mathrm{~nm}$ intervals, this gave a milling thickness of $48 \pm 8.6 \mathrm{~nm}$ (mean $\pm \mathrm{SD}$ ); for the series cut at $15 \mathrm{~nm}$ intervals, the milling thickness was measured as $14.1 \pm 3.1 \mathrm{~nm}$ (mean \pm SD). It is evident in the serial
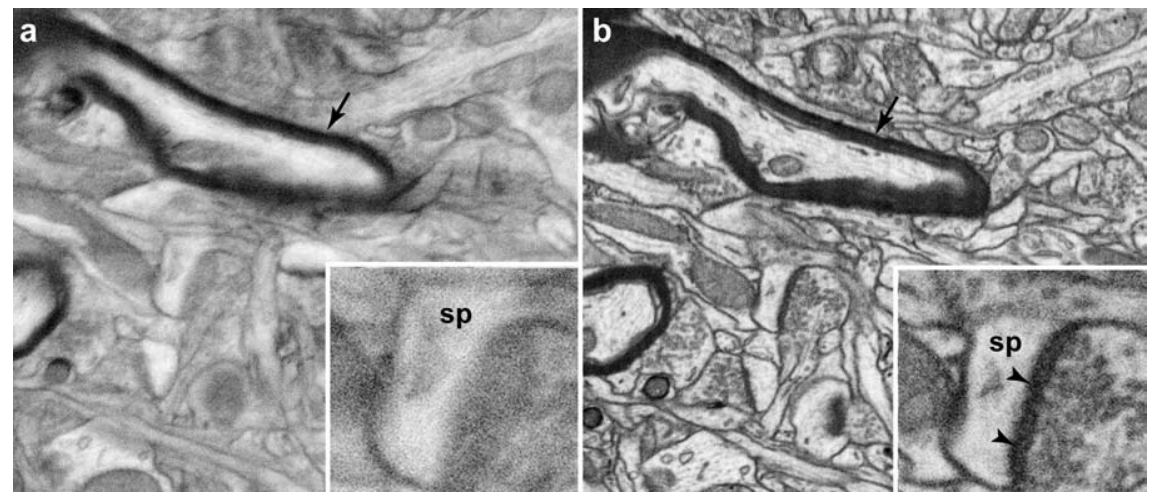

Figure 3. $a$, Reverse-contrast backscattered electron micrograph of a region of neuropil with a resolution of $4 \mathrm{~nm} / \mathrm{pixel}$. The electron beam energy was $5 \mathrm{keV}$. The image shows limited contrast between the stained membranes and intracellular space. This effect appears to blur the image. $\boldsymbol{b}$, Reverse-contrast micrograph of the same region of the imaging face that was imaged in $\boldsymbol{a}$. All the imaging parameters are the same, except that the electron beam energy has been lowered to $2 \mathrm{keV}$. In this image, the membrane contrast is improved and the depth of imaging is decreased. Arrows in both images indicate a myelinated axon. The inset images in both panels show a dendritic spine head (sp), but only in $\boldsymbol{b}$ can the cluster of vesicles be seen along with the presynaptic and postsynaptic densities (arrowheads).

images (supplemental movie 1, available at www.jneurosci.org as supplemental material) that milling thickness is not entirely consistent throughout the stack, although it is not clear why this occurred.

An analysis of neural connectivity requires the identification of the synapses. The parameters that we chose to collect this image series allowed for the identification of single vesicles, making it possible to see the neuronal connections. Moreover, morphological differences between identified synapses indicated that both asymmetric (presumed glutamatergic) and symmetric (presumed GABAergic) synapses could also be distinguished (Fig. 4; supplemental movie 1 , available at www.jneurosci.org as supplemental material). For asymmetric synapses, vesicles were round, and their lumens appeared clear and unstained (Fig. 4). These were present in axonal boutons making contacts that were asymmetric, with the postsynaptic density being thicker than the presynaptic. This was in contrast to symmetric synapses, where the vesicles were ovoid, smaller than the asymmetric type, with the axonal boutons displaying presynaptic and postsynaptic densities that were of a similar thickness (Fig. 4). In our other studies in this region of the neocortex, all these symmetric synapses were immunopositive for GABA (Knott et al., 2002).

\section{Discussion}

Visualizing the complete wiring and connections within a neural circuit is a realistic challenge with today's EM technology. Using TEM, serial sections can be collected, stained, and imaged, and the sec- tions revisited numerous times at any magnification (Harris et al., 2006). For the moment, much of this process needs to be done by hand and is not without pitfalls. The serial block-face scanning SEM approach (Denk and Horstmann, 2004), although removing sections that are discarded, is capable of collecting aligned series of images automatically, and with large fields of view that are not possible with currently available TEMs. The diamond knife is well suited to sectioning these large block faces. However, imaging this surface at a resolution suitable to see synapses means that significant energy is delivered to the block-face, resulting in changes in the resin's structure (i.e., crosslinking); the increased hardness making it difficult to continue the sectioning process with the knife (Denk and Horstmann, 2004).

These technical limitations led us to explore the FIB milling technology with the aim of producing a series of images in which all the neurites and their connections could be identified. The resulting images only required limited alignment. Moreover, we found that imaging at a resolution that allowed us to see the axons, dendrites, and synapses did not change the properties of the resin that would interfere with the milling process. This meant that cutting with the ion beam, although limited in area, is possible regardless of the parameters of the imaging beam. Excessive cross-linking of the embedding resin does not appear to affect the milling beam and is not noticeable in the final image series.

We were able to mill a surface area of $>10,000 \mu \mathrm{m}^{2}$, which we then reduced to 
$900 \mu \mathrm{m}^{2}$ when we collected the final image series. The maximum possible area that can be milled, however, is limited. The largest length along which the ion beam can scan is $800 \mu \mathrm{m}$. This theoretical width to the imaging window means that the beam would be deflected far from the vertical. Ions at the outer edges would not be localized to a circular spot, but an ellipse, with a larger surface area, resulting in fewer ions impacting the resin per unit area, and decreasing their milling effect. Here we milled a region $120 \mu \mathrm{m}$ wide without seeing any detrimental effects at the windows' edges. The milling depth is also limited. The beam is focused on the upper edge of the block face giving the best imaging surface in the upper regions. Further down, the face the beam is less focused, reducing the milling effect and decreasing the chance that ions will be able to eject molecules away from the block.

Milling the final $900 \mu \mathrm{m}^{2}$ face took $75 \mathrm{~s}$, which was insignificant for the short series of acquired images. When images are required over much larger surfaces, however, the milling time would become significant, as well as the possibility of charge buildup over the imaged surface. Although the initial block is coated with platinum, providing a conductive layer surrounding the imaged regions, this may become less effective when large areas are prepared.

Despite the milling time, image acquisition time is the main limiting factor for large volumes. Each image is generated as a result of the electron beam scanning the block surface, and a balance needs to be reached between fast acquisition time, high resolution, and adequate signal to generate images with the suitable contrast. Using a $4 \mathrm{~nm} /$ pixel magnification and dwell time of $100 \mu \mathrm{s} /$ pixel, each image was acquired in $4 \mathrm{~min}$, and the total stack in $>12$ h. Although significant improvements in the dwell time could reduce the total imaging time, we were not able to achieve this and also maintain an image quality with sufficient contrast.

The technique allowed us to see and follow all the neurites in the image series and recognize their synaptic connections. However, there was certain variation in milling depth from image to image, through the stack. This was considerably reduced when milling thinner layers, but it is not clear why, so this needs further investigation. For the series of images taken here, the inconsistency in milling depth does not appear to cause problems in following the neurites through the neu-
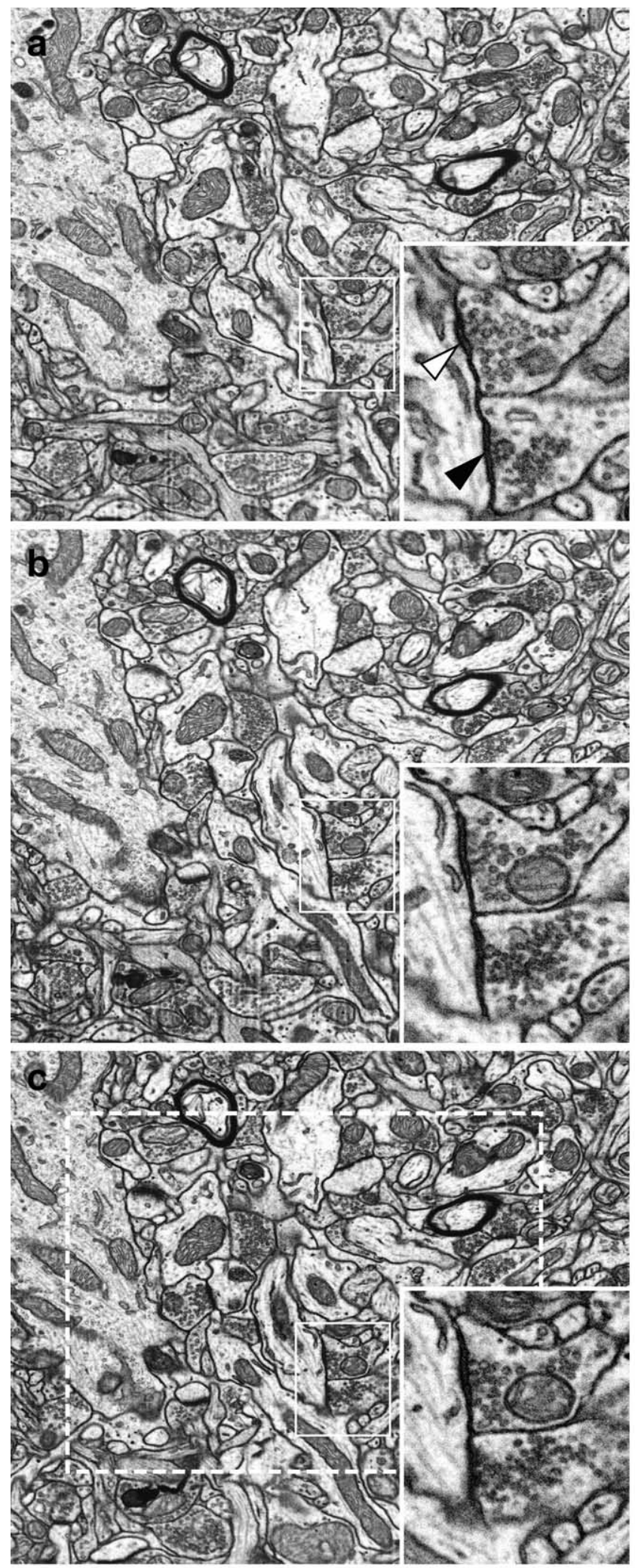

Figure 4. $\boldsymbol{a}-\boldsymbol{c}$, Serial scanning electron micrographs taken from the imaging face each time that $40 \mathrm{~nm}$ of resin had been milled away. The beam voltage was $2 \mathrm{keV}$, the beam current was $0.4 \mathrm{nA}$, and the dwell time was $100 \mu \mathrm{s}$. The magnification was set at $4 \mathrm{~nm} / \mathrm{pixel}$, giving a field width of $8.4 \mu \mathrm{m}$. In each image, the inset shows a digitally magnified region that is indicated with the white square showing a bouton making an asymmetric synapase (white arrowhead) and a bouton making a symmetric synapse (black arrowhead). The dotted line in c indicates a portion of the field of view that was selected from the stack of 120 images that is shown in supplemental movie 1 (available at www.jneurosci.org as supplemental material). 
ropil. We could also identify both the symmetric and asymmetric synapses. However, because some of the neuronal processes, such as dendritic spine necks, can be as thin as $40 \mathrm{~nm}$ (Sorra and Harris, 2000), it may be necessary to mill thinner layers. Layers of $15 \mathrm{~nm}$, although possible, and increasing the ability to automatically segment all of the structures, would also increase the number of images needed.

In conclusion, this study shows that ion beam milling, combined with blockface scanning, can be used to generate serial images through substantial volumes of neuropil in the adult mammalian brain. These image series had sufficient contrast and resolution to identify all the axons and dendrites and their synaptic contacts. However, as an approach to collecting images on a large scale through volumes of nervous tissue that may even contain complete neural circuits, this block-face scanning technique needs further improvements. For the moment, scanning beam technology is limited in its ability to mill large surfaces, and imaging across these areas at high resolution would require considerable time. Nevertheless, the method is able to identify synapses, and the whole process of milling and image acquisition can be completely automated without relying on manual collection, staining, and imaging of large series of sections.

\section{References}

Anderson JC, Douglas RJ, Martin KA, Nelson JC (1994) Map of the synapses formed with the dendrites of spiny stellate neurons of cat visual cortex. J Comp Neurol 341:25-38.

Denk W, Horstmann H (2004) Serial block-face scanning electron microscopy to reconstruct three-dimensional tissue nanostructure. PLoS Biol 2:e329.

Fiala JC, Harris KM (2001) Cylindrical diameters method for calibrating section thickness in serial electron microscopy. J Microsc 202:468-472.

Gray EG (1959) Axo-somatic and axo-dendritic synapses of the cerebral cortex: an electron microscope study. J Anat 93:420-433.

Harris KM, Perry E, Bourne J, Feinberg M, Ostroff L, Hurlburt J (2006) Uniform serial sectioning for transmission electron microscopy. J Neurosci 26:12101-12103.
Heymann JA, Hayles M, Gestmann I, Giannuzzi LA, Lich B, Subramaniam S (2006) Sitespecific 3D imaging of cells and tissues with a dual beam microscope. J Struct Biol 155:63-73.

Knott GW, Quairiaux C, Genoud C, Welker E (2002) Formation of dendritic spines with GABAergic synapses induced by whisker stimulation in adult mice. Neuron 34:265-273.

Shepherd GM, Harris KM (1998) Threedimensional structure and composition of CA3->CA1 axons in rat hippocampal slices: implications for presynaptic connectivity and compartmentalization. J Neurosci 18:8300-8310.

Sorra KE, Harris KM (2000) Overview on the structure, composition, function, development, and plasticity of hippocampal dendritic spines. Hippocampus 10:501-511.

White EL, Rock MP (1980) Three-dimensional aspects and synaptic relationships of a Golgiimpregnated spiny stellate cell reconstructed from serial thin sections. J Neurocytol 9:615-636.

White JG, Southgate E, Thomson JN, Brenner S (1986) The structure of the nervous system of the nematode Caenorhabditis elegans. Philos Trans R Soc Lond A 314:1-340. 